\title{
Some Relations Between the Values of a Function and its First Derivative at $n$ Abscissa Points
}

\author{
By Robert E. Huddleston
}

Abstract. For a polynomial, $P$, of degree $2 n-2$, there exists a relation between the values of $P$ and the values of its first derivative, $P^{\prime}$, at the $n$ abscissa points $x_{1}, \cdots, x_{n}$,

$$
\sum_{i=1}^{n}\left[a_{i} P\left(x_{i}\right)+b_{i} P^{\prime}\left(x_{i}\right)\right]=0 .
$$

Replacing $P$ by a differentiable function $y$ yields

$$
\sum_{i=1}^{n}\left[a_{i} y\left(x_{i}\right)+b_{i} y^{\prime}\left(x_{i}\right)\right]=E(y, x) .
$$

These relations are obtained and the error function $E(y, x)$ is given explicitly.

1. Introduction. For a polynomial, $P_{2 n-2}$, of degree $2 n-2$, there exists a relation between the values of $P_{2 n-2}$ and its first derivative at the $n$ distinct abscissas $x_{1}, \cdots, x_{n}$. If $n=2$ and $x_{2}-x_{1}=h$, the relation is

$$
2 P_{2}\left(x_{1}\right)+h P_{2}^{\prime}\left(x_{1}\right)=2 P_{2}\left(x_{2}\right)-h P_{2}^{\prime}\left(x_{2}\right) \text {. }
$$

If we impose the condition that $x_{i}=x_{1}+(j-1) h, j=1,2, \cdots, n$ (fixed step-size case), then at least for low-order $n$, there is a reasonably straightforward method for determining such relations [1, p. 247]. If we are given a function $y$ which is differentiable, how much error do we incur by using the polynomial relation for $y$ ? For $n=2$, we are asking what the term $E(x, y)$ is in the relation

$$
2 y\left(x_{1}\right)+h y^{\prime}\left(x_{1}\right)=2 y\left(x_{2}\right)-h y^{\prime}\left(x_{2}\right)+E(x, y) .
$$

For the fixed step-size case and for $n \leqq 4$, these relations are in the literature $\left[1\right.$, p. 247]. However, for the case in which the $x_{i}$ are not evenly spaced, no such results are available. It is the purpose of this paper to derive such relations along with the corresponding error relations.

2. Method. Let $y_{i} \equiv y\left(x_{i}\right)$ and $y_{i}^{\prime} \equiv y^{\prime}\left(x_{i}\right), j=1, \cdots, n$. We shall use a method which in essence is the derivation of Hermite interpolation, given the data $\left(x_{1}, y_{1}, y_{1}^{\prime}\right), \cdots,\left(x_{n-1}, y_{n-1}, y_{n-1}^{\prime}\right),\left(x_{n}, y_{n}^{\prime}\right)$. The lack of data for $y_{n}$ causes most of the difficulties. However, if one is familiar with the derivation of Hermite interpolation [3, p. 192], the derivation of the following relations will be recognized as an exercise in drudgery. The determination of the relation for $E(x, y)$ is not so straightforward. Hence, we shall first determine the polynomial $P_{2 n-2}$ such that $P_{2 n-2}\left(x_{i}\right)=y_{i}, i=1$,

Received June 25, 1970.

AMS 1970 subject classifications. Primary 65L05; Secondary 26A75.

Key words and phrases. Ordinary differential equations, Runge-Kutta, error estimation for Runge-Kutta, one-step methods for ordinary differential equations, polynomials. 
$\cdots, n-1$, and $P_{2 n-2}^{\prime}\left(x_{i}\right)=y_{i}^{\prime}, i=1, \cdots, n$. Then, we will evaluate the polynomial at $x_{n}$ and find $E\left(x_{n}, y\right)$ such that

$$
y\left(x_{n}\right)=P_{2 n-2}\left(x_{n}\right)+E\left(x_{n}, y\right) .
$$

3. Derivation of $P_{2 n-2}$. Following the idea of Hermite interpolation, we search for $P_{2 n-2}$ of the form

$$
P_{2 n-2}(x)=\sum_{i=1}^{n-1} \alpha_{i}(x) y_{i}+\sum_{i=1}^{n} \beta_{i}(x) y_{i}^{\prime}
$$

where

$$
\begin{aligned}
& \alpha_{i}\left(x_{i}\right)=\delta_{i j}, \quad j=1, \cdots, n-1, \quad \text { and } \beta_{i}\left(x_{i}\right)=0, \quad j=1, \cdots, n-1, \\
& \alpha_{i}^{\prime}\left(x_{i}\right)=0, \quad j=1, \cdots, n, \quad \beta_{i}^{\prime}\left(x_{i}\right)=\delta_{i j}, \quad j=1, \cdots, n \text {. }
\end{aligned}
$$

Let

$$
l_{i}(x)=\frac{1}{A_{i}} \prod_{i=1 ; i \ngtr i}^{n-1}\left(x-x_{i}\right)
$$

where

$$
A_{i}=\prod_{i=1 ; i \neq i}^{n-1}\left(x_{i}-x_{i}\right)
$$

The $\alpha_{i}$ can then be represented by

$$
\alpha_{i}(x)=A_{i}^{2} l_{i}^{2}(x)\left[\gamma_{i} x^{2}+\delta_{i} x+\eta_{i}\right]
$$

where the $\gamma_{i}, \delta_{i}$, and $\eta_{i}$ are to be determined by the conditions $\alpha_{i}\left(x_{i}\right)=1, \alpha_{i}^{\prime}\left(x_{i}\right)=$ $\alpha_{i}^{\prime}\left(x_{n}\right)=0$. After much algebraic manipulation we arrive at

$$
\begin{aligned}
\gamma_{i} & =\frac{1}{A_{i}^{2}\left(x_{n}-x_{i}\right)}\left[l_{i}^{\prime}\left(x_{i}\right)+\frac{l_{i}^{\prime}\left(x_{n}\right)\left[\left(x_{n}-x_{i}\right) l_{i}^{\prime}\left(x_{i}\right)-1\right]}{l_{i}\left(x_{n}\right)+\left(x_{n}-x_{i}\right) l_{i}^{\prime}\left(x_{n}\right)}\right], \\
\delta_{i} & =-2 A_{i}^{-2} l_{i}^{\prime}\left(x_{i}\right)-2 \gamma_{i} x_{i}, \\
\eta_{i} & =A_{i}^{-2}+2 A_{i}^{-2} x_{i} l_{i}^{\prime}\left(x_{i}\right)+x_{i}^{2} \gamma_{i} .
\end{aligned}
$$

Since we are interested in evaluating $P_{2 n-2}(x)$ at $x_{n}$, we find

$$
\alpha_{i}\left(x_{n}\right)=-l_{i}^{2}\left(x_{n}\right)\left[\sum_{i=1}^{n-1} \frac{1}{x_{n}-x_{i}}\right]^{-1}\left[\sum_{i=1 ; j \neq i}^{n} \frac{1}{x_{i}-x_{i}}\right] .
$$

In like manner the $\beta_{i}$, for $i=1, \cdots, n-1$, may be represented by

$$
\beta_{i}(x)=A_{i}^{2} l_{i}^{2}(x)\left[a_{i} x^{2}+b_{i} x+c_{i}\right]
$$

where the $a_{i}, b_{i}$, and $c_{i}$ are to be determined by $\beta_{i}\left(x_{i}\right)=\beta_{i}^{\prime}\left(x_{n}\right)=0$ and $\beta_{i}^{\prime}\left(x_{i}\right)=1$. This yields

$$
a_{i}=-\frac{1}{2 A_{i}^{2}}\left[\frac{1}{x_{n}-x_{i}}+\frac{l_{i}^{\prime}\left(x_{n}\right)}{l_{i}(x)+\left(x_{n}-x_{i}\right) l_{i}^{\prime}\left(x_{n}\right)}\right]
$$


and

$$
\begin{aligned}
& b_{i}=A_{i}^{-2}-2 a_{i} x_{i}, \\
& c_{i}=x_{i}\left[a_{i} x_{i}-A_{i}^{-2}\right] .
\end{aligned}
$$

The term $\beta_{n}(x)$ is determined separately and yields

$$
\beta_{n}(x)=\frac{l_{n}^{2}(x)}{2 l_{n}^{\prime}\left(x_{n}\right)}
$$

Combining these relations for the $\beta_{i}$ and evaluating at $x_{n}$, we have

$$
\beta_{i}\left(x_{n}\right)=\frac{1}{2} l_{i}^{2}\left(x_{n}\right)\left[\sum_{i=1}^{n-1} \frac{1}{x_{n}-x_{i}}\right]^{-1}, \quad j=1, \cdots, n .
$$

From (3.1), (3.2), and (3.3), we have

$$
\begin{aligned}
& P_{2 n-2}\left(x_{n}\right) \\
& \quad=\left(\sum_{i=1}^{n-1} \frac{1}{x_{n}-x_{j}}\right)^{-1}\left\{-\sum_{i=1}^{n-1}\left[l_{i}^{2}\left(x_{n}\right)\left(\sum_{i=1 ; i \neq i}^{n} \frac{1}{x_{i}-x_{j}}\right) y_{i}\right]+\frac{1}{2} \sum_{i=1}^{n} l_{i}^{2}\left(x_{n}\right) y_{i}^{\prime}\right\} .
\end{aligned}
$$

4. Determination of the Error. We now wish to determine $E\left(y, x_{n}\right)$ in the relation

$$
y\left(x_{n}\right)=P_{2 n-2}\left(x_{n}\right)+E\left(y, x_{n}\right) .
$$

Let

$$
\phi(x)=2\left(x_{n}-x\right) l_{n}^{2}(x) l_{n}^{\prime}\left(x_{n}\right)+l_{n}^{2}(x) .
$$

Then

$$
\begin{aligned}
\phi\left(x_{i}\right) & =\delta_{n i}, & j & =1, \cdots, n, \\
\phi^{\prime}\left(x_{i}\right) & =0, & j & =1, \cdots, n .
\end{aligned}
$$

Let $F$ be defined by

$$
F(x)=y(x)-P_{2 n-2}(x)-\phi(x)\left[y\left(x_{n}\right)-P_{2 n-2}\left(x_{n}\right)\right] .
$$

Then $F$ has the properties

$$
\begin{aligned}
F\left(x_{i}\right) & =0, \quad j=1, \cdots, n, \quad \text { and } \\
F^{\prime}\left(x_{i}\right) & =0, \quad j=1, \cdots, n .
\end{aligned}
$$

Hence, $F$ has at least $2 n$ zeroes ( $n$ double zeroes) in the smallest interval $J$ containing $x_{1}, \cdots, x_{n}$. [Note that $l_{n}^{\prime}\left(x_{n}\right)$ is nonzero provided that $x_{n}$ lies outside the smallest interval, $I$, containing $x_{1}, \cdots, x_{n-1}$.] Applying Rolle's theorem $(2 n-1)$ times, we may state that $F^{(2 n-1)}$ has at least one zero in $J$. Let $\zeta$ be such a zero. From (4.2),

$$
F^{(2 n-1)}(x)=y^{(2 n-1)}(x)-P_{2 n-2}^{(2 n-1)}(x)-\phi^{(2 n-1)}(x)\left[y\left(x_{n}\right)-P_{2 n-2}\left(x_{n}\right)\right] .
$$

But $P_{2 n-2}^{(2 n-1)}(x) \equiv 0$ since the degree of $P_{2 n-2}(x)=2 n-2$. If one notes that $\phi(x)$ is a polynomial of degree $2 n-1$ with leading coefficient $-2 A_{n}^{-2} l_{n}^{\prime}\left(x_{n}\right)$, then it is clear that

$$
\phi^{(2 n-1)}(x)=-2(2 n-1) ! A_{n}^{-2} l_{n}^{\prime}\left(x_{n}\right)
$$


Hence,

$$
0=F^{(2 n-1)}(\zeta)=y^{(2 n-1)}(\zeta)+2(2 n-1) ! A_{n}^{-2} l_{n}^{\prime}\left(x_{n}\right)\left[y\left(x_{n}\right)-P_{2 n-2}\left(x_{n}\right)\right] .
$$

Thus, we have established the following:

THEOREM. If $y \in C^{(2 n-1)}[J]$, then

$$
y\left(x_{n}\right)=P_{2 n-2}\left(x_{n}\right)-\frac{A_{n}^{2} y^{(2 n-1)}(\zeta)}{2(2 n-1) ! l_{n}^{\prime}\left(x_{n}\right)},
$$

where $\zeta \in J$ and $x_{n} \notin I$.

5. Relations Between $y\left(x_{i}\right)$ and $y^{\prime}\left(x_{i}\right)$. From (3.4) and (4.4) and use of the identity

$$
l_{i}^{\prime}(x)=l_{i}(x) \sum_{i=1 ; j ; i}^{n-1} \frac{1}{x_{i}-x_{i}},
$$

we arrive at the desired relation between $y\left(x_{i}\right)$ and $y^{\prime}\left(x_{i}\right), j=1, \cdots, n$ :

$$
\sum_{i=1}^{n}\left\{\prod_{i=1 ; i \neq i}^{n-1}\left(\frac{x_{n}-x_{i}}{x_{i}-x_{i}}\right)^{2}\left[y_{i}^{\prime}-\left(\sum_{i=1 ; i \neq i}^{n} \frac{2}{x_{i}-x_{j}}\right) y_{i}\right]\right\}=-E\left(x_{n}, y\right)
$$

where

$$
E\left(x_{n}, y\right)=-\frac{\prod_{i=1}^{n-1}\left(x_{n}-x_{i}\right)^{2} y^{(2 n-1)}(\zeta)}{2(2 n-1) ! \sum_{i=1}^{n-1}\left(\frac{1}{x_{n}-x_{i}}\right)} .
$$

For the special case of even step-size $\left(x_{i}=x_{1}+(j-1) h, j=1, \cdots, n\right)$, the above relations reduce to

$$
\sum_{i=1}^{n}\left\{\left(\begin{array}{c}
n-1 \\
i-1
\end{array}\right)\left[h y_{i}^{\prime}-\left(\sum_{i=1 ; j \neq i}^{n} \frac{2}{i-j}\right) y_{i}\right]\right\}=-E\left(x_{n}, y\right),
$$

where

$$
E\left(x_{n}, y\right)=-\frac{[(n-1) !]^{2}}{2(2 n-1) !} h^{2 n-1} y^{(2 n-1)}(\zeta)
$$

6. An Alternate Approach. If we had given the data $\left(x_{1}, y_{1}, y_{1}^{\prime}\right), \cdots,\left(x_{n-1}\right.$, $\left.y_{n-1}, y_{n-1}^{\prime}\right),\left(x_{n}, y_{n}\right)$ and determined the polynomial

$$
Q_{2 n-2}(x)=\sum_{i=1}^{n} q_{i}(x) y_{i}+\sum_{i=1}^{n-1} r_{i}(x) y_{i}^{\prime}
$$

such that

$$
\begin{array}{ll}
Q_{2 n-2}\left(x_{i}\right)=y_{i}, & i=1, \cdots, n, \\
Q_{2 n-2}^{\prime}\left(x_{i}\right)=y_{i}^{\prime}, & i=1, \cdots, n-1,
\end{array}
$$

we would have had a much easier task in deriving expressions for the $q_{i}$ and $r_{i}$. We could have then determined $e(x, y)$ such that

$$
y(x)=Q_{2 n-2}(x)+e(x, y) .
$$


Having done this, we could differentiate (6.1), evaluate it at $x_{n}$, and have the desired relationship between $y\left(x_{i}\right)$ and $y^{\prime}\left(x_{i}\right)$ for $j=1, \cdots, n$. However, the proof that $e(x, y)$ can be differentiated as a function of $x$ and the resulting differentiation represent a considerable task. From (6.1) and (6.2), we may write

$$
y_{n}=\sum_{i=1}^{n-1}\left(-\frac{q_{i}^{\prime}\left(x_{n}\right)}{q_{n}^{\prime}\left(x_{n}\right)} y_{i}\right)+\sum_{i=1}^{n-1}\left(-\frac{r_{i}^{\prime}\left(x_{n}\right)}{q_{n}^{\prime}\left(x_{n}\right)} y_{i}^{\prime}\right)+\frac{y^{\prime}\left(x_{n}\right)}{q_{n}^{\prime}\left(x_{n}\right)}-\frac{e^{\prime}\left(x_{n}, y\right)}{q_{n}^{\prime}\left(x_{n}\right)}
$$

which we may compare with

$$
y_{n}=\sum_{i=1}^{n-1} \alpha_{i}\left(x_{n}\right) y_{i}+\sum_{i=1}^{n} \beta_{i}\left(x_{n}\right) y_{i}^{\prime}+E\left(x_{n}, y\right) .
$$

Choosing $y$ to be an arbitrary polynomial of degree exactly $2 n-2$, we note that the error terms are zero. Noting also that (1) the coefficients $\alpha_{i}, \beta_{i}, q_{i}$, and $r_{i}$ are independent of $y$, and (2) that the relation between the values of a polynomial of degree $2 n-2$ and its derivatives at $n$ distinct abscissa points is unique, we arrive at the conclusion that

$$
\begin{aligned}
& \alpha_{i}\left(x_{n}\right)=-\frac{q_{i}^{\prime}\left(x_{n}\right)}{q_{n}^{\prime}\left(x_{n}\right)} \text { and } \beta_{i}\left(x_{n}\right)=-\frac{r_{i}^{\prime}\left(x_{n}\right)}{q_{n}^{\prime}\left(x_{n}\right)}, \\
& \qquad \text { for } i=1, \cdots, n-1 \text { and } \beta_{n}\left(x_{n}\right)=\frac{1}{q_{n}^{\prime}\left(x_{n}\right)} .
\end{aligned}
$$

But then from (6.3) and (6.4), we conclude that

$$
e_{n}^{\prime}\left(x_{n}, y\right)=-q_{n}^{\prime}\left(x_{n}\right) E\left(x_{n}, y\right) .
$$

7. Applications to Differential Equations. In order to approximate the solution of the initial-value problem

$$
y^{\prime}(x)=f(x, y(x)), \quad y(a)=A,
$$

one often uses a one-step scheme of the form

$$
y_{n+1}=y_{n}+h \Phi\left(x_{n}, y_{n}, h_{n}\right), \quad y_{0}=A,
$$

where $h_{n}=x_{n+1}-x_{n}$. The local truncation error, $\tau_{n}$, in proceeding from $x_{n}$ to $x_{n+1}$, is defined by

$$
\tau_{n}=Z\left(x_{n+1}\right)-y_{n+1}
$$

where $Z(x)$ is given by

$$
Z^{\prime}(x)=f(x, Z(x)), \quad Z\left(x_{n}\right)=y_{n} .
$$

Using the relationship (5.3) for $Z$ with $n=2$, we have

$$
2 Z\left(x_{n}\right)+h Z^{\prime}\left(x_{n}\right)=2 Z\left(x_{n+1}\right)-h Z^{\prime}\left(x_{n+1}\right)+O\left(h^{3}\right),
$$

which, from (7.3) and (7.4), yields

$$
2 y_{n}+h f\left(x_{n}, y_{n}\right)=2\left[\tau_{n}+y_{n+1}\right]-h f\left(x_{n+1}, \tau_{n}+y_{n+1}\right)+O\left(h^{3}\right) .
$$

Using a Taylor series expansion, we have

$$
f\left(x_{n+1}, \tau_{n}+y_{n+1}\right)=f\left(x_{n+1}, y_{n+1}\right)+f_{y}\left(x_{n+1}, y_{n+1}\right) \tau_{n}+O\left(\tau_{n}^{2}\right) .
$$


Substituting this in (7.5), we have

$$
\tau_{n}=\left[y_{n}-y_{n+1}\right]+\frac{1}{2} h\left[f\left(x_{n}, y_{n}\right)+f\left(x_{n+1}, y_{n+1}\right)\right]+h f_{y}\left(x_{n+1}, y_{n+1}\right) \tau_{n}+O\left(h \tau_{n}^{2}\right) .
$$

If this estimate is used with a one-step method having local truncation error of order $h^{2}$ (for example, Euler's method), then the last two terms of (7.6) are of the order $h^{3}$ and $h^{5}$ and, hence, are negligible with respect to the local truncation error. Thus, we have the estimate

$$
\tau_{n}=\left[y_{n}-y_{n+1}\right]+\frac{1}{2} h\left[f\left(x_{n}, y_{n}\right)+f\left(x_{n+1}, y_{n+1}\right)\right] .
$$

Such estimates are derivable for one-step methods of higher order through the use of relation (5.1). The extensions are not straightforward and constitute the subject of [2]. It is important to note that the quantities needed for the estimate (7.7) are those normally calculated in a one-step procedure and thus require no additional function evaluations. This property is characteristic of estimates derivable from (5.1) (see [2]) and thus results in error estimates which are very inexpensive with respect to computer time. In particular, the estimates can replace the time consuming process, so often used with Runge-Kutta, of carrying two simultaneous calculations with step-sizes $h$ and $2 h$ and comparing the answers for step-size control.

Sandia Laboratories

Numerical Applications Division 8321

Livermore, California 94550

1. F. Ceschino \& J. Kuntzmann, Numerical Solution of Initial Value Problems, PrenticeHall, Englewood Cliffs, N. J., 1966. MR 33 \#3465.

2. R. E. HUDDLESTON, Variable-Step Truncation Error Estimates for Runge-Kutta Methods of Order 4 or Less, Report \#DC-70-261, Sandia Laboratories, Livermore, California.

3. E. IsaAcson \& H. B. Keller, Analysis of Numerical Methods, Wiley, New York, 1966. MR 34 \#924. 